\title{
Different types of spin currents in the comprehensive materials database of nonmagnetic spin Hall effect
}

\author{
Yang Zhang ${ }^{1,2,3,8}$, Qiunan $\mathrm{Xu}^{1,8}$, Klaus Koepernik ${ }^{2}$, Roman Rezaev $\mathbb{D}^{2}$, Oleg Janson ${ }^{2}$, Jakub Železný ${ }^{4}$, Tomáš Jungwirth ${ }^{4,5}$, \\ Claudia Felser $\left(\mathbb{D}^{1,6}\right.$, Jeroen van den Brink ${ }^{2,7}$ and Yan Sun ${ }^{1 凶}$
}

Spin Hall effect (SHE) has its special position in spintronics. To gain new insight into SHE and to identify materials with substantial spin Hall conductivity (SHC), we performed high-precision high-throughput ab initio calculations of the intrinsic SHC for over 20,000 nonmagnetic crystals. The calculations revealed a strong relationship between the magnitude of the SHC and the crystalline symmetry, where a large SHC is typically associated with mirror symmetry-protected nodal line band structures. This database includes 11 materials with an SHC comparable to or even larger than that of Pt. Materials with different types of spin currents were additionally identified. Furthermore, we found that different types of spin current can be obtained by rotating applied electrical fields. This improves our understanding and is expected to facilitate the design of new types of spin-orbitronic devices.

npj Computational Materials (2021)7:167; https://doi.org/10.1038/s41524-021-00635-0

\section{INTRODUCTION}

Even though the extrinsic spin Hall effect (SHE) was predicted almost 50 years ago $^{1,2}$, the SHE did not receive extensive attention until the last decade, after theoretical studies of its intrinsic mechanism ${ }^{3,4}$ and its experimental observation ${ }^{5-7}$. The SHE gives rise to an electrical current to generate a transverse spin current ${ }^{1,2,8}$, and vice versa ${ }^{9-11}$. The advantages of strong SHE materials, which are of central importance for the detection, generation, and manipulation of spin currents suggested the necessity of performing large-scale screening to identify the most suitable materials for spintronics devices. Experimentally, however, such large-scale screening would be highly impractical, as quantitative determination of the spin Hall conductivity (SHC) by electrical measurement requires integrating each material separately into a complex multicomponent microscopic transport device ${ }^{9-11}$. In contrast, theoretically and computationally the situation is in principle much more straightforward. An additional advantage of high-throughput calculations is that they can provide further insight into the physics of the SHE and suggest general guidelines for designing new SHE materials.

In general, the SHE has two origins: an extrinsic contribution from scattering and intrinsic contributions from electronic band structures. In this study, we focus only on the intrinsic contribution for two reasons. First, the intrinsic contribution is typically dominant in systems with strong spin-orbit coupling $(\mathrm{SOC})^{8}$. Second, the SHE can be accurately predicted theoretically as long as the calculation of the electronic structure is sufficiently precise $e^{3,4,8,12-14}$. Contrary to this, the extrinsic contributions are much more difficult to predict and strongly depend on the type of scattering. In many cases, the calculated intrinsic SHE is in good qualitative agreement with the experimental measurements $s^{8,15}$. For example, the large intrinsic SHC of $\mathrm{Pt}^{16-19}$ or the predicted sign change of the $\mathrm{SHC}$ from $\mathrm{Pt}$ to $\mathrm{Ta}$ were experimentally observed ${ }^{20,21}$. Therefore, this database is anticipated to be helpful for selecting new materials with a spinto-charge conversion, even if the predicted values are not expected to be precisely reproducible in experiments. Apart from the SHC, other parameters could also be used to determine the usefulness of material for spin-to-charge conversion, in particular the spin Hall angle $(\mathrm{SHA})^{8,22}$. Although we did not attempt to evaluate these parameters in this study, we note that the charge conductivity is straightforward to measure experimentally; thus, the SHAs can be easily obtained from our calculations for materials for which the conductivity is experimentally known.

\section{RESULTS AND DISCUSSION Workflow}

We developed automatic Wannier function (WF) generating code to enable us to conduct high-throughput simulations and calculations. The workflow of the procedure we followed to build a collection of suitable materials for inclusion in our database is shown in Fig. 1. Most of the materials we studied are in the ICSD database ${ }^{23}$, which contains details of real materials that have been experimentally characterized. In addition, we also considered computational materials from the Materials Project ${ }^{24,25}$ (MP), because they were extracted from alloys or similar structures on the basis of experimentally known materials and could thus possibly be synthesized. Owing to the limited accuracy of the density functional theory (DFT) for strongly correlated systems, we did not consider computational materials from the MP that contain $\mathrm{V}, \mathrm{Mn}, \mathrm{Fe}, \mathrm{Co}, \mathrm{Ni}, \mathrm{N}, \mathrm{O}, \mathrm{F}, \mathrm{Cl}$, or f-electron atoms. This is because these materials are often strongly correlated and the predicted structures may thus be less accurate (see Fig. 1). We did not remove the materials that are possibly strongly correlated from the ICSD database, as it is almost impossible to accurately estimate the strength of these correlations from high-throughput calculations. Furthermore, generalized gradient approximation (GGA)-level calculations would be useful even for those materials characterized by strong correlations. However, we did remove all

\footnotetext{
${ }^{1}$ Max Planck Institute for Chemical Physics of Solids, 01187 Dresden, Germany. ${ }^{2}$ Leibniz Institute for Solid State and Materials Research, 01069 Dresden, Germany. ${ }^{3}$ Department of Physics, Massachusetts Institute of Technology, Cambridge, MA 02139, USA. ${ }^{4}$ Institute of Physics, Czech Academy of Sciences, Cukrovarnicka 10, 16200 Praha 6, Czech Republic. ${ }^{5}$ School of Physics and Astronomy, University of Nottingham, NG7 2RD Nottingham, United Kingdom. ${ }^{6}$ Center for Nanoscale Systems, Faculty of Arts and Sciences, Harvard University, 11 Oxford Street, LISE 308, Cambridge, MA 02138, USA. ${ }^{7}$ Institut fur Theoretische Physik and Wurzburg-Dresden Cluster of Excellence ct.qmat, Dresden University of Technology, 01062 Dresden, Germany. ${ }^{8}$ These authors contributed equally: Yang Zhang, Qiunan Xu. ${ }^{\circledR}$ email: ysun@cpfs.mpg.de
} 


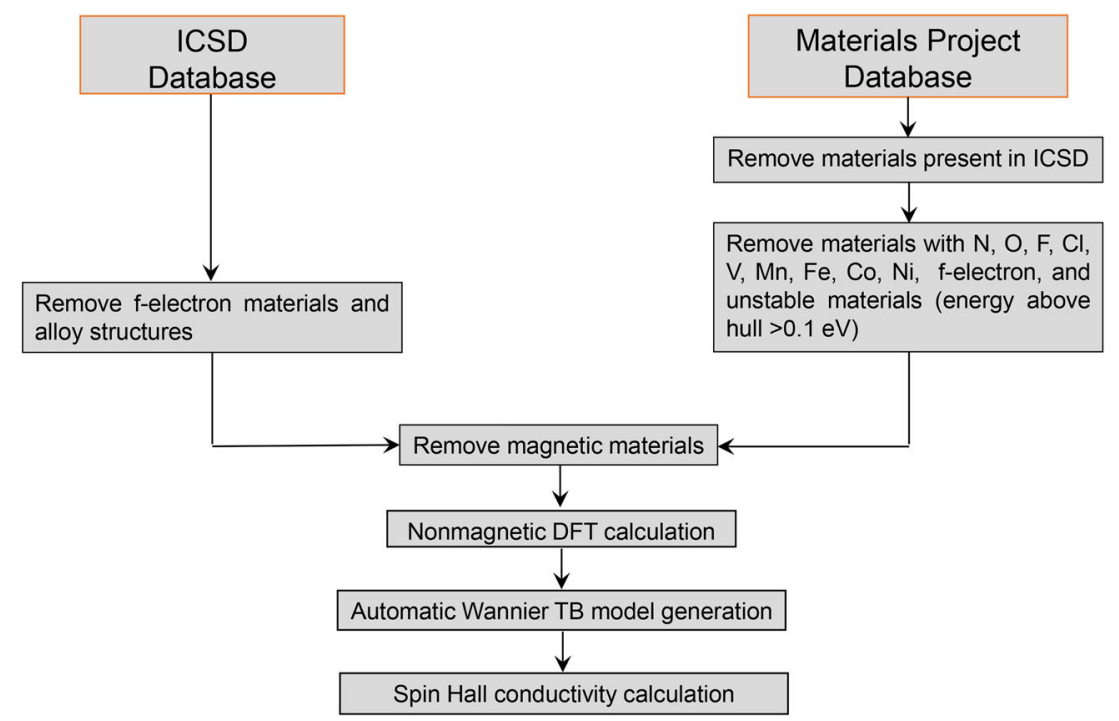

Fig. 1 Workflow to obtain the dataset of materials for the high-throughput calculations of intrinsic SHC.

f-electron systems. We then used the MP database to identify materials that were likely to be magnetic. After excluding these materials, 17,682 real materials from the ICSD database and 2486 computational materials from the MP remained.

These lattice structures were then loaded into the full-potential local-orbital minimum-basis code of FPLO for DFT calculations ${ }^{26,27}$. We then projected the Bloch wavefunctions into highly symmetric atomic-orbital-like WFs, and generated the corresponding tightbinding model Hamiltonians. The symmetry of the tight-binding model Hamiltonian is essential to determine the SHC accurately. Based on the tight-binding model Hamiltonian, the intrinsic SHCs were computed by the Kubo formula approach ${ }^{4,12,14}$. The accuracy of the calculated SHCs was doubly checked by the symmetry analysis ${ }^{28,29}$. All of the SHC calculations were based on DFT calculations for nonmagnetic materials. A few of the materials we considered could have been magnetic; however, the SHC calculation for these materials would not be accurate. We thus checked the magnetic states of materials with large SHC $(>500(\hbar)$ e) $(\mathrm{S} / \mathrm{cm})$ ) using spin-polarized DFT calculations to eliminate the remaining magnetic materials.

\section{Data overview}

The distribution of the calculated SHC for the full set of materials is shown in Fig. 2. (Details of each of these compounds can be found on our website). Among the $>20,000$ materials, we identified only 1048 with an SHC larger than $500(\hbar / e)(S / c m)$, and only 169 for which the value was above $1000(\hbar / e)(S / \mathrm{cm})$. We identified eleven materials with an SHC comparable to or even larger than that of Pt: IrN, $\ln _{5} \mathrm{Bi}_{3}, \mathrm{Tl}_{3} \mathrm{Ru}, \mathrm{Pt}_{3} \mathrm{Rh}, \mathrm{CuPt}_{7}, \mathrm{LiPt}_{7}, \mathrm{Bi}_{2} \mathrm{OsAu}, \mathrm{Bi}_{3} \mathrm{In}_{4} \mathrm{~Pb}$, $\mathrm{HgOsPb}_{2}$, Lilr, and $\mathrm{PtRh}_{3}$. Of these materials, $\ln _{5} \mathrm{Bi}_{3}, \mathrm{CuPt}_{7}, \mathrm{LiPt}_{7}$, and Lilr were already synthesized in experiments (details appear in the SI). The compound with the largest SHC is $\mathrm{IrN}$, which has an SHC larger than $2900(\hbar / e)(\mathrm{S} / \mathrm{cm})$ in $\sigma_{z x}^{y}$; however, we note that this is for a theoretically proposed phase of $\operatorname{IrN}^{30,31}$. The next largest is $\operatorname{In}_{5} \mathrm{Bi}_{3}$, which, unlike the other compounds with large SHC that contain $5 d$ elements, is a pure $p$-orbital metal with an SHC above $2500(\hbar / e)(S / \mathrm{cm})$ in the $\sigma_{x y}^{z}$ component. Apart from the recordbreaking in $\ln _{5} \mathrm{Bi}_{3}$, the high-throughput calculations identified four other non-transition metal compounds $\left(\operatorname{lnBi}, \ln _{2} B i, \ln _{5} B i_{2} P b\right.$, and the element $\mathrm{TI}$ ) with a large SHE. The fact that only a handful of the 20,000 crystals we studied has an SHC larger than that of Pt and none has a significantly higher value, suggests that we may have approached the realistic limit of the intrinsic SHC in our calculations.

\section{Giant SHC}

To analyze the origin of the large SHC, we considered the spin Berry curvature (SBC) distribution in the Brillouin zone (BZ) of the top 12 compounds with $\mathrm{SHC}>2000(\hbar / e)(\mathrm{S} / \mathrm{cm})$. We discovered that in most of these materials, the large SHC is associated with nodal lines protected by the mirror symmetry, see Fig. 2c. The nodal lines are one-dimensional band crossings, which exist in the absence of SOC protected by the mirror (or other) symmetry ${ }^{32}$. With SOC, the nodal lines are split, which gives rise to a large SBC if the nodal lines cross the Fermi level. Particulars for two selected materials, $\ln _{5} \mathrm{Bi}_{3}$ and $\mathrm{Pt}$, are shown in Fig. 3 and those of the others are included in the SI. The giant SHC in $\mathrm{In}_{5} \mathrm{Bi}_{3}$ is related to the mirror planes $m_{100}$ and $m_{110}$, which cause two independent nodal lines in the $k_{x}=0$ and $k_{x}+k_{y}=0$ planes, respectively. In addition to these mirror symmetry-protected nodal lines, $\ln _{5} \mathrm{Bi}_{3}$ also contains special PT symmetry-protected nodal rings away from high-symmetry planes. It is clear from Fig. 3a that the hotspots of SBC are dominated by these nodal lines.

A similar symmetry analysis of $\mathrm{Pt}$ indicated that also in this wellknown SHE material, the large SHC originates from mirror symmetry: it protects the nodal rings of which the SBC distribution is shown in Fig. 3c. We also created a local integral of the SBC in the spheres with centers of high-symmetry points of $L$ and $X$ and diameters of $1 / 10$ of the reciprocal lattice vectors. Interestingly, the contribution to the SBCs by the high-symmetry points of $L$ and $X$ is $<15 \%$ of the contribution by the nodal lines. Even if the SBC was very large around the two high-symmetry points of $L$ and $X$, their volumes were much smaller than those of the nodal lines. This suggests the important advantage of the nodal rings in the context of SHE: their significant dispersion in energy space indicates the existence of a large possibility of crossing the Fermi level and contributing strongly to the SHC. This understanding clearly explains the strong SHC in the classical martial Pt, which was ignored in the past.

The SBC analysis for the other ten compounds also showed the importance of nodal line band structures, see SI. In 9 of the 12 materials (IrN, $\mathrm{Tl}_{3} \mathrm{Ru}, \mathrm{Pt}_{3} \mathrm{Rh}, \mathrm{CuPt}_{7}, \mathrm{LiPt}_{7}, \mathrm{Bi}_{2} \mathrm{OsAu}, \mathrm{Bi}_{3} \mathrm{In}_{4} \mathrm{~Pb}$, $\mathrm{HgOsPb}_{2}$, Lilr, and $\mathrm{PtRh} 3$ ) the main contributions to the $\mathrm{SBC}$ originate from mirror symmetry-protected nodal lines. As mentioned above, in $\ln _{5} \mathrm{Bi}_{3}$ the main contributions originate from nodal lines protected by both mirror symmetry and PT symmetry. In Lilr and IrN both, mirror symmetry-protected nodal lines and other hotspots contribute (see the SI for more details). 

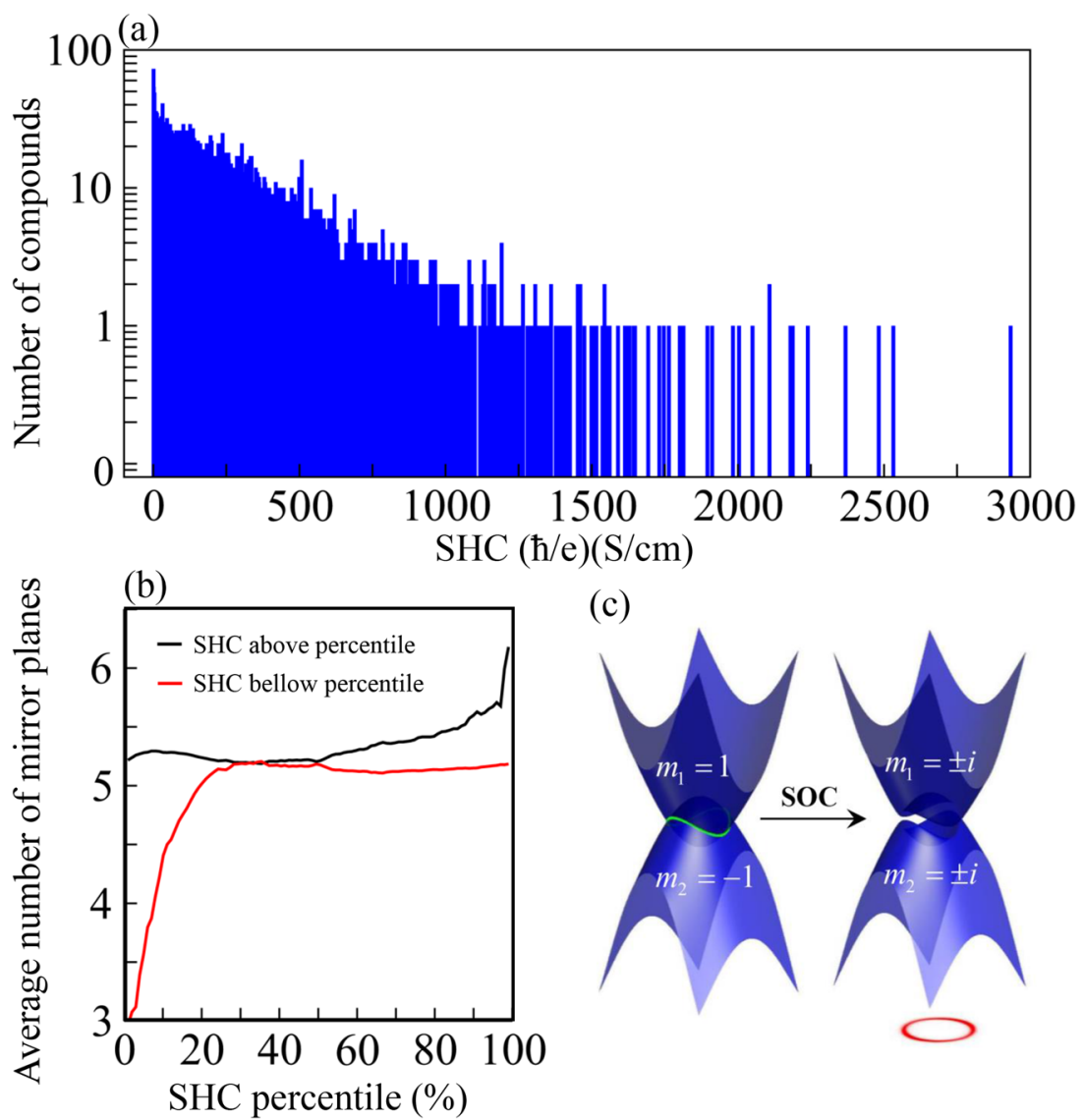

(c)

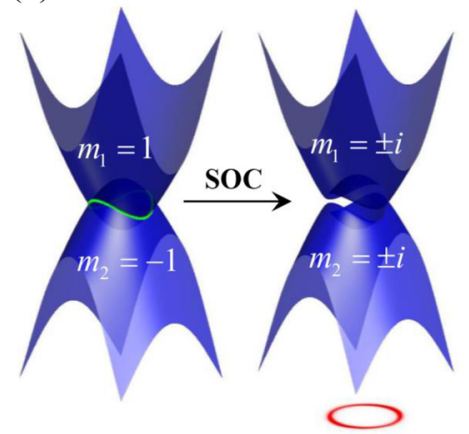

Fig. 2 Calculated intrinsic SHC. a Histogram of SHC values for all calculated materials. b Average number of mirror planes for materials with an SHC above a percentile of mirror planes and below a percentile of mirror planes as a function of the percentile. For example, the red curve shows the average number of mirror planes for $80 \%$ of the materials with the lowest $\mathrm{SHC}$, whereas the black curve shows the average for the top $20 \%$ of the materials. c Illustration of the nodal line mechanism. The splitting of the bands from SOC results in a large SBC along the original nodal line, as illustrated by the red circle.

In addition to materials with large native SHCs, it is also possible to obtain a strong SHE by tuning the chemical potential in a given compound if it has large peak values near the Fermi level. We found that the addition of a dopant to fill the holes can increase the SHC from $\sim 1200$ to $\sim 2300(\hbar / e)(S / c m)$ in $\mathrm{IrN}_{2}$, from $\sim 1100$ to $\sim 2000(\hbar / e)(\mathrm{S} / \mathrm{cm})$ in NalrPb, and from 400 to $2000(\hbar / e)(\mathrm{S} / \mathrm{cm})$ in $\mathrm{TaAsPb}_{2}$. Electron doping can increase the $\mathrm{SHC}$ from $\sim 800$ to $\sim 2000(\hbar / e)(\mathrm{S} / \mathrm{cm})$ in BeReB and from 200 to $1500(\hbar / e)(\mathrm{S} / \mathrm{cm})$ in $\mathrm{TaBe}_{3}$, etc. Details can be found on the website. Using the energydependent SHC for guidance, intrinsic or extrinsic doping by tuning the chemical potential to the peak value can increase the $\mathrm{SHC}$ to meet the experimental requirements.

Microscopic analysis suggests that mirror symmetry has an important role in generating a large SHC. Similarly, we found that materials with many mirror planes have a larger SHC on average than materials without mirror planes. The mean value of the SHC in materials with at least three mirror planes is $28 \%$ larger than the mean value in materials without mirror planes. In materials without mirror planes, the mean value is 212 at the $95 \%$ confidence interval $(189,233)$, whereas in materials with at least three mirror planes, the mean value is 271 at the $95 \%$ confidence interval $(264,277)$. The confidence intervals were estimated using the empirical bootstrapping method. The effect is the most pronounced in materials with large SHC. Among all the materials, $16 \%$ of materials have less than three mirror planes, among the top 500 , the corresponding percentage is only $8 \%$, and among the top 50 materials, it is only $2 \%$. This is also illustrated in Fig. 2 b, which shows that the materials with the largest SHC have more mirror planes on average than the other materials. We note that for the statistical analysis we excluded insulating materials (materials with a density of states at the Fermi level of lower than 0.01). Our results show that mirror symmetry is one of the key factors that influence the magnitude of the SHC. Although it does not exert any effect on average, it seems to be crucial for generating a very large SHC.

Importantly, an analogous relation between the SHC and mirror symmetry is likely to exist in other effects that have a similar origin to the intrinsic SHE, such as the anomalous Hall effect, or antidamping spin-orbit torque. Thus, our results have quite a fundamental significance: it shows that the symmetry determines not only the presence or absence of transport phenomena, as previously thought, but can also have a strong influence on their magnitude.

\section{Different types of spin currents}

In commonly used materials, the symmetry of the SHE is such that the spin polarization of the spin current is perpendicular to both the spin current and the charge current. This is not a general rule, however. In materials with low crystalline symmetry, other types of spin current are allowed ${ }^{33}$. This includes a spin current that flows in the transverse direction to the charge current, similar to normal SHE but has spin polarization along the direction of the spin current flow, the electric field, or longitudinal spin currents, that is, spin currents that flow in the same direction as the charge current, as illustrated in Fig. 4. These types of spin currents could allow for new functionalities in spintronic devices; however, they have not received much experimental attention because materials 

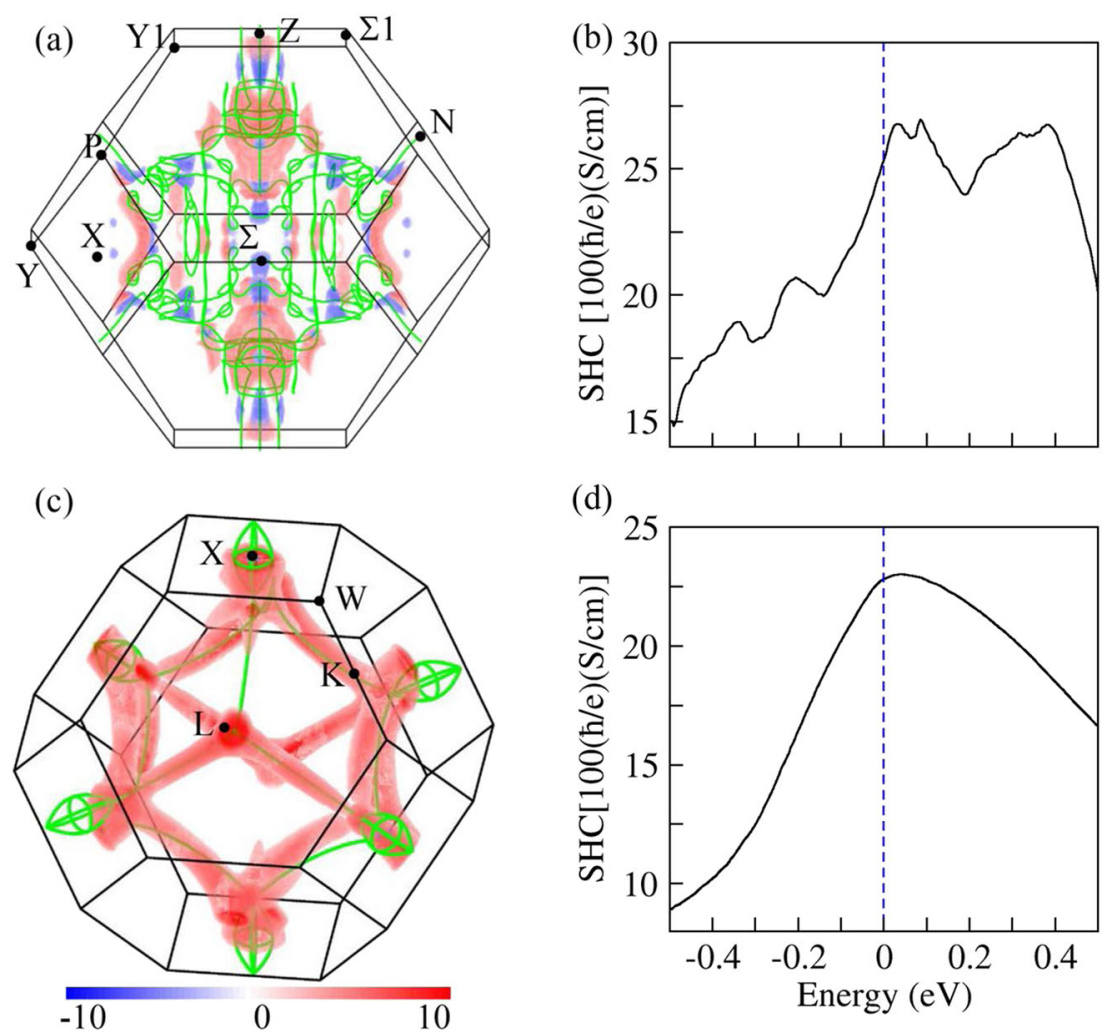

Fig. 3 Microscopic analysis for selected materials. SBC and nodal line distributions for (a) $\ln _{5} \mathrm{Bi}_{3}$ with point group $4 / \mathrm{mmm}$, and (c) $\mathrm{Pt}$ with point group $m \overline{3} m$, respectively. The green lines represent nodal lines. The color bar is in arbitrary units. Fermi-level-dependent SHC for (b) $\ln _{5} \mathrm{Bi}_{3}$ and (d) Pt, respectively.

in which they are allowed are quite rare. A theoretical screening to identify promising materials is thus essential. We note that the SHE normally refers to a spin current flowing in the transverse direction to the charge current; thus, it is not clear whether longitudinal spin currents should be referred to as SHE. Nevertheless, we stress that the longitudinal spin currents have the same origin as the conventional SHE.

The existence and magnitude of such unusual symmetry spin currents, which are not perpendicular to the flow of the spin current, the spin polarization, and the electrical field depend on the orientation of the applied electrical field. For an electric field applied in high-symmetry directions, such spin currents are allowed only in several Laue groups. For an electric field applied in an arbitrary direction, we find that unusual symmetry spin currents can be present in all the Laue groups, except for those with $m \overline{3} m$ symmetry. All the materials from our database that have spin currents with an unusual symmetry larger than $100(\hbar / e)$ $(\mathrm{S} / \mathrm{cm})$ are listed in the SI.

The SHE, which flows in the transverse direction to the charge current and has a spin polarization along the spin current flow direction, is of great interest for spin-orbit torques in heavy metalferromagnet bilayer systems (or other similar systems) ${ }^{34}$ (see Fig. 4b). In these structures, the SHE from the heavy metal layer flows into the ferromagnetic layer and thus exerts a torque on the magnetization $^{35}$. For scalability, it is preferential to utilize a ferromagnet with a perpendicular magnetic anisotropy (PMA). However, for deterministic field-free switching of PMA systems, it is necessary to have a spin current with spin polarization perpendicular to the interface (and thus parallel to the direction of the spin current flow). This is not allowed by the symmetry of the materials commonly used in these systems, such as Pt; thus, materials with lower symmetry are needed. Our calculations revealed that a large SHC with spin polarization along the spin current can occur, but is relatively rare. Considering the arbitrary direction of the electric field, we found 58 materials with an SHC above $500(\hbar / e)(\mathrm{S} / \mathrm{cm})$ with the largest value, $900(\hbar / e)(\mathrm{S} / \mathrm{cm})$, exhibited by $\mathrm{BiTe}_{3}$. When we considered the electric field along the high-symmetry directions, we found only two materials with values above $500(\hbar / e)(\mathrm{S} / \mathrm{cm}): \mathrm{Ni}_{2} \mathrm{P}_{6} \mathrm{~W}_{4}$ and $\mathrm{Ba}_{2} \mathrm{C}_{4} \mathrm{~S}_{4} \mathrm{~N}_{4}$.

The longitudinal spin currents in ferromagnetic systems were experimentally studied, where the origin of these currents lies in the ferromagnetic order. In our database, we identified a number of materials that exhibit large longitudinal spin currents with their spin polarization parallel or perpendicular to the spin current flow, as shown in Fig. 4c. In the case of an electric field with an arbitrary direction, we found seven materials with longitudinal spin currents larger than $500(\hbar / e)(S / \mathrm{cm})$ with the largest being $\sim 1010(\hbar / e)(S / \mathrm{cm})$ in Lilr. For an electric field along with the high-symmetry directions, the largest longitudinal spin current is $\sim 610(\hbar / e)(\mathrm{S} / \mathrm{cm})$ at $\sigma_{z z}^{z}$ in $\mathrm{P}_{7} \mathrm{Ru}_{12} \mathrm{Sc}_{2}$. The longitudinal spin current in nonmagnetic crystals may offer a new platform for the study and utilization of the spin current in nonmagnetic materials.

Another type of spin current is one that flows in the transverse direction to the electric field and has a spin polarization along the direction of the electric field, as shown in Fig. 4d. The study of the non-orthogonal $\mathrm{SHE}^{36,37}$ via manipulation of the crystalline symmetry opens an avenue to study the spin current and spinorbital torque based on nonmagnetic materials. In our database, we identified 67 materials with this type of spin current larger than $500(\hbar / e)(\mathrm{S} / \mathrm{cm})$.

Our calculations revealed that the origin of the large SHC is usually associated with mirror symmetry-protected nodal lines in the band structure, which results in a strong correlation between the crystalline symmetry and magnitude of the SHC. This suggests 
(a)

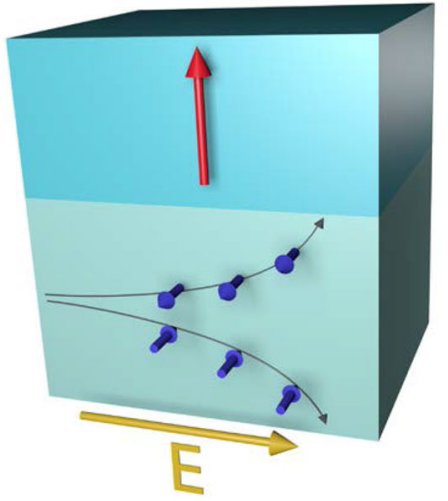

(c)

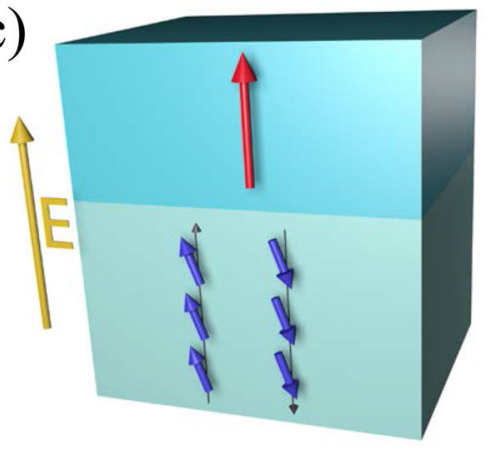

(b)

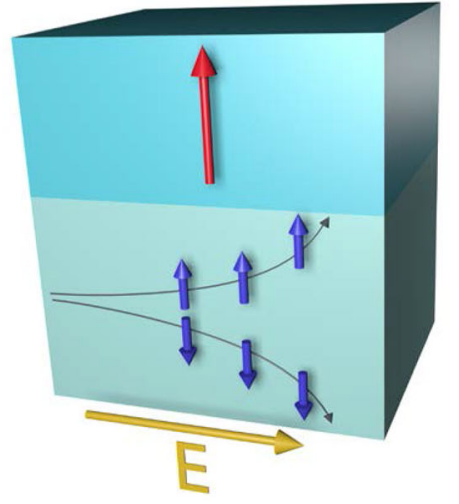

(d)

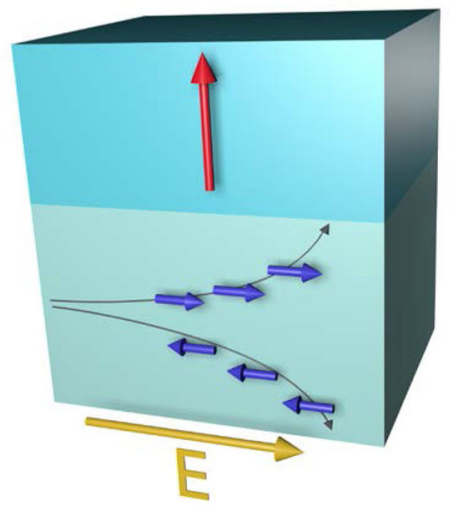

Fig. 4 Heterojunction of ferromagnets and non-magnets with four types of spin current included in this database. The top and bottom layers are ferromagnetic and non-ferromagnetic, respectively. a Traditional SHE with the spin polarization of the spin current perpendicular to both the spin current and the charge current. b Spin current flowing in the direction transverse to the electric field with the spin polarization orientated in the direction of the flow. c Longitudinal spin current flowing in the direction of the electric field. $\mathbf{d}$ Spin current flowing in the direction transverse to the electric field with the spin polarization existing along the direction of the electric field.

that for the design of new SHC materials, it is beneficial to consider high-symmetry materials with a large number of mirror planes. In addition, we determined that, apart from the obvious requirement for the presence of heavy elements with a strong $\mathrm{SOC}$, the presence of $5 d$ transition metal elements is advantageous, but not decisive. We identified a number of promising spinto-charge conversion materials, including 169 materials with an SHC above $1000(\hbar / e)(\mathrm{S} / \mathrm{cm}), 11$ materials with an SHC comparable or even larger than that of the existing record holder, $\mathrm{Pt}$, and materials in which the symmetry of the SHE is lower thus allowing for different types of spin currents. These different types of spin current can also be obtained by adjusting the orientation of the applied electrical field. With these general design principles on the one hand, and the specific information on each separate compound, on the other hand, our high-throughput database is a powerful tool for the experimental design of spintronic devices.

\section{METHODS}

\section{Autmatic WF Setup}

The ab initio calculations were carried out on the basis of DFT by using the FPLO $\operatorname{code}^{26}$. The exchange and correlation energies were considered in the $\mathrm{GGA}^{27}$. The $k$-point grid in the DFT calculation was set to $12 \times 12 \times 12$, and the criterion for total energy convergency below $10^{-6} \mathrm{eV}$. To perform the SHC, we projected the Bloch wavefunctions to atomic-orbital-like WFs by an automatic procedure. To ensure the Wannier projection is automatic and accurate, we set the constraint of the mean error between the DFT and tight binding below $0.02 \mathrm{eV}$ in the energy window of $E_{f}-2.0 \mathrm{eV}$ to $\mathrm{E}_{f}+2.0 \mathrm{eV}$. The symmetry of the WFs is well restored by the FPLO DFT code, where these WFs are directly mapped from the atomic orbitals without a maximum localization process.

We design the automatic Wannierization workflow by fixing two crucial parameters: (1) Wannier orbital basis set, (2) projected energy window for Wannier tight-binding model. Initially, the $s$ orbitals of alkaline-earth metal elements, $d$ orbitals of transition metal elements, and $s+p$ orbitals of $p$ block elements are selected as the minimum Wannier orbital basis. After the band structure calculations, we further calculate the weights of the selected Wannier orbitals in the DFT Kohn-Sham wavefunctions and check whether the Wannier orbitals are disentangled from the inner valence orbitals.

When there is a global gap between the selected Wannier orbital basis set and the inner valence orbitals, we fix the bottom of the projected energy window in the gap. We then choose the up projected energy window by every $0.1 \mathrm{eV}$ step and construct the Wannier tight-binding models correspondingly. The chosen Wannier orbitals are accepted until the mean absolute error between the DFT and Wannier band structures around the Fermi level is smaller than $0.02 \mathrm{eV}$.

When the minimum Wannier orbital basis set is entangled with the inner valence orbitals, we employ the maximum Wannier orbital basis set including all the local orbitals used in DFT calculations. Such a maximum Wannier orbital basis set automatically ensures the highly accurate Wannier tight-binding model, at the expense of relatively large Hilbert space, namely, 18 orbitals per atom. Because FPLO is a DFT code based on a localized basis, it makes this procedure convenient ${ }^{38}$.

\section{SHC calculation}

Starting from the tight-binding model Hamiltonian, the SHC was calculated by using the linear response Kubo formula approach with a constant $\Gamma$ 


$$
\begin{aligned}
& \text { approximation }^{8,14} \text { : } \\
& \begin{aligned}
\sigma_{i j}^{k} & =e \int_{B z} \frac{d \vec{k}}{(2 \pi)^{3}} \sum_{n} f_{n \vec{k}} \Omega_{n, i j}^{S, k}(\vec{k}), \Omega_{n, i j}^{S, k}(\vec{k}) \\
& =-2 / m \sum_{n^{\prime} \neq n} \frac{\left\langle n \vec{k}\left|J_{i}^{k}\right| n^{\prime} \vec{k}\right\rangle\left\langle n^{\prime} \vec{k}\left|v_{j}\right| n \vec{k}\right\rangle}{\left(E_{n \vec{k}}-E_{n^{\prime}} \vec{k}\right)^{2}+\Gamma^{2}},
\end{aligned}
\end{aligned}
$$

where $f_{n \vec{k}}$ is the Fermi-Dirac distribution for the $n$th band, $J_{i}^{k}=$ $\frac{1}{2}\left\{v_{i}, \quad s_{k}\right\}$ is the spin current operator with the spin operator s, velocity operator $v_{i}$, and $i, j, k=x, y, z$. Further, $|n \vec{k}\rangle$ is the eigenvector for the Hamiltonian $H$ at eigenvalue $E_{n} \vec{k}$. In addition, $\Omega_{n, j}^{S, k}(\vec{k})$ is referred to as the SBC for the $n$th band at point $\vec{k}$, as an analogy to the ordinary Berry curvature. We calculated the intrinsic contribution which is independent of any scattering and that we only include very small broadening $\Gamma=$ $0.1 \mathrm{meV}$ for numerical reasons. After checking the convergence of the $k$ grid for 2,000 randomly chosen compounds, we find that the change in the SHE is below $5 \%$ by increasing the $k$-grid from $150 \times 150 \times 150$ to $250 \times 250 \times 250$. The changes in the cases with $\mathrm{SHC}>500(\hbar / e)(\mathrm{S} / \mathrm{cm})$ are even smaller. Here, we chose $250 \times 250 \times 250$ k-grid for all SHC calculations. One point we need to emphasize is that the calculation of the SHC needs to consider the real position of Wannier orbitals in the phase factors; otherwise, the calculated SHC would differ markedly for some compounds. All the calculated results were doubly verified by symmetry analysis.

\section{DATA AVAILABILITY}

All data generated and/or analyzed during this study are included in this article, its Supplementary Information file, and the corresponding website: https://shc-db.ifwdresden.de/index. The data are available from the corresponding author upon reasonable request.

\section{CODE AVAILABILITY}

All code used to calculate the presented results is available from the corresponding author upon reasonable request.

Received: 10 April 2021; Accepted: 16 September 2021; Published online: 12 October 2021

\section{REFERENCES}

1. Dyakonov, M. I. \& Perel, V. I. Possibility of orienting electron spins with current. $Z h$. Eksp. Teor. Fiz. 13, 657 (1971).

2. Dyakonov, M. I. \& Perel, V. I. Current-induced spin orientation of electrons in semiconductors. Phy. Lett. A 36, 459 (1971).

3. Murakami, S., Nagaosa, N. \& Zhang, S.-C. Dissipationless quantum spin current at room temperature. Science 301, 1348 (2003).

4. Sinova, J. et al. Universal intrinsic spin hall effect. Phy. Rev. Lett. 92, 126603 (2004).

5. Kato, Y. K., Myers, R. C., Gossard, A. C. \& Awschalom, D. D. Observation of the spin Hall effect in semiconductors. Science 306, 1910 (2004).

6. Wunderlich, J., Kaestner, B., Sinova, J. \& Jungwirth, T. Experimental observation of the spin-hall effect in a two-dimensional spin-orbit coupled semiconductor system. Phy. Rev. Lett. 94, 047204 (2005).

7. Day, C. Two groups observe the spin hall effect in semiconductors. Phys. Today 58, 17 (2005).

8. Sinova, J., Valenzuela, S. O., Wunderlich, J., Back, C. \& Jungwirth, T. Spin Hall effects. Rev. Mod. Phys. 87, 1213 (2015).

9. Saitoh, E., Ueda, M., Miyajima, H. \& Tatara, G. Conversion of spin current into charge current at room temperature: inverse spin-Hall effect. Appl. Phys. Lett. 88, 182509 (2006)

10. Valenzuela, S. O. \& Tinkham, M. Direct electronic measurement of the spin Hall effect. Nature 44, 176 (2006)

11. Zhao, H., Loren, E. J., Driel, H. M. V. \& Smirl, A. L. S. Coherence control of hall charge and spin currents. Phy. Rev. Lett. 96, 246601 (2006).

12. Guo, G. Y., Yao, Y. \& Niu, Q. Ab initio calculation of the intrinsic spin Hall effect in semiconductors. Phy. Rev. Lett. 94, 226601 (2005).

13. Freimuth, F., Blügel, S. \& Mokrousov, Y. Anisotropic spin Hall effect from first principles. Phy. Rev. Lett. 105, 246602 (2010).
14. Xiao, D., Chang, M.-C. \& Niu, Q. Berry phase effects on electronic properties. Rev. Mod. Phys. 82, 1959-2007 (2010).

15. Hoffmann, A. Spin hall effects in metals. IEEE Trans. Magn. 49, 5172 (2013).

16. Kimura, T., Otani, Y., Sato, T., Takahashi, S. \& Maekawa, S. Room-temperature reversible spin hall effect. Phy. Rev. Lett. 98, 156601 (2007).

17. Seki, T. et al. Giant spin Hall effect in perpendicularly spin-polarized FePt/Au devices. Nat. Mat. 7, 125 (2008).

18. Isasa, M., Villamor, E., Hueso, L. E., Gradhand, M. \& Casanova, F. Temperature dependence of spin diffusion length and spin Hall angle in Au and Pt. Phy. Rev. B 92, 019905 (2015).

19. Zhu, L., Zhu, L., Sui, M., Ralph, D. C. \& Buhrman, R. A. Variation of the giant intrinsic spin Hall conductivity of Pt with carrier lifetime. Sci. Adv. 5, eaav8025 (2019).

20. Tanaka, T. et al. Intrinsic spin Hall effect and orbital Hall effect in $4 d$ and $5 d$ transition metals. Phy. Rev. B 77, 165117 (2008).

21. Hahn, C., Loubens, G. D., Klein, O. \& Viret, M. Comparative measurements of inverse spin Hall effects and magnetoresistance in YIG/Pt and YIG/Ta. Phy. Rev. B 87, 174417 (2013).

22. Wang, L. et al. Giant room temperature interface spin hall and inverse spin hall effects. Phys. Rev. Lett. 116, 196602 (2016).

23. Hellenbrandt, M. The Inorganic Crystal Structure Database (ICSD)-present and future. Crystallogr. Rev. 10, 17 (2004).

24. Jain, A. et al. Commentary: the Materials Project: a materials genome approach to accelerating materials innovation. APL Mater. 1, 011002 (2013).

25. Jain, A. et al. Commentary: the materials project: a materials genome approach to accelerating materials innovation. APL Mater. 1, 011002 (2013).

26. Koepernik, K. \& Eschrig, H. Full-potential nonorthogonal local-orbital minimumbasis band-structure scheme. Phys. Rev. B 59, 1743 (1999).

27. Perdew, J. P., Burke, K. \& Ernzerhof, M. Generalized gradient approximation made simple. Phys. Rev. Lett. 77, 3865 (1996).

28. Kleiner, W. H. Space-time symmetry of transport coefficients. Phys. Rev. 142, 318 (1966).

29. Seemann, M., Kodderitzsch, D., Wimmer, S. \& Ebert, H. Symmetry-imposed shape of linear response tensors. Phys. Rev. B 92, 155138 (2015).

30. Li, W. The structural phase transition and elastic properties of IrN under high pressure from first-principles calculations. J. Alloy. Compd. 537, 216-220 (2012).

31. Rached, H. et al. Structural stabilities, elastic, and electronic properties of iridium mononitride: a first-principles study. Phase Transit. 84, 269-283 (2011).

32. Fang, C., Weng, H., Dai, X. \& Fang, Z. Topological nodal line semimetals. Chin. Phys. B 25, 117106 (2016).

33. Wimmer, S., Seemann, M., Chadova, K., Ködderitzsch, D. \& Ebert, H. Spin-orbitinduced longitudinal spin-polarized currents in nonmagnetic solids. Phys. Rev. $B$ 92, 041101 (2015).

34. MacNeill, D. et al. Control of spin-orbit torques through crystal symmetry in WTe2/ferromagnet bilayers. Nat. Phys. 13, 300 (2016).

35. Manchon, A. et al. Current-induced spin-orbit torques in ferromagnetic and antiferromagnetic systems. Rev. Mod. Phys. 91, 035004 (2019).

36. Song, P. et al. Coexistence of large conventional and planar spin Hall effect with long spin diffusion length in a low-symmetry semimetal at room temperature. Nat. Mater. 19, 292-298 (2020).

37. MacNeill, D. et al. Control of spin-orbit torques through crystal symmetry in WTe2/ferromagnet bilayers. Nat. Phys. 13, 300-305 (2017).

38. $\mathrm{Xu}, \mathrm{Q}$. et al. Comprehensive scan for nonmagnetic Weyl semimetals with nonlinear optical response. npj Comput. Mater. 6, 1-7 (2020).

\section{ACKNOWLEDGEMENTS}

This work was financially supported by the ERC Advanced Grant no. 291472 'Idea Heusler', ERC Advanced Grant no. 742068-TOPMAT, Deutsche Forschungsgemeinschaft DFG under SFB 1143, and EU FET Open RIA Grant no. 766566 grant (ASPIN). This work was performed in part at the Center for Nanoscale Systems (CNS), a member of the National Nanotechnology Coordinated Infrastructure Network (NNCl), which is supported by the National Science Foundation under NSF award no. 1541959. CNS is part of Harvard University. Most of our calculations are carried on Cobra cluster of MPCDF, Max Planck Society. J.Ž. acknowledges funding from the Czech Science Foundation Grant no. 19-18623Y and support from the Institute of Physics of the Czech Academy of Sciences and the Max Planck Society through the Max Planck Partner Group programme.

\section{AUTHOR CONTRIBUTIONS}

Y.Z., Q.X., J.Ž., and Y.S. carried out all calculations. K.K. made the code for WF projection. R.R., O.J., and J. van den B. setup the database webpage. T.J., C.F., J. van den B., and Y.S. supervised this work. Y.Z. and Q.X. wrote the first version of the manuscript. All authors discussed the results and commented on the paper. 


\section{FUNDING}

Open Access funding enabled and organized by Projekt DEAL.

\section{COMPETING INTERESTS}

The authors declare no competing interests

\section{ADDITIONAL INFORMATION}

Supplementary information The online version contains supplementary material available at https://doi.org/10.1038/s41524-021-00635-0.

Correspondence and requests for materials should be addressed to Yan Sun

Reprints and permission information is available at http://www.nature.com/ reprints

Publisher's note Springer Nature remains neutral with regard to jurisdictional claims in published maps and institutional affiliations.
Open Access This article is licensed under a Creative Commons Attribution 4.0 International License, which permits use, sharing, adaptation, distribution and reproduction in any medium or format, as long as you give appropriate credit to the original author(s) and the source, provide a link to the Creative Commons license, and indicate if changes were made. The images or other third party material in this article are included in the article's Creative Commons license, unless indicated otherwise in a credit line to the material. If material is not included in the article's Creative Commons license and your intended use is not permitted by statutory regulation or exceeds the permitted use, you will need to obtain permission directly from the copyright holder. To view a copy of this license, visit http://creativecommons. org/licenses/by/4.0/.

(c) The Author(s) 2021, corrected publication 2021 